\title{
Sheath-Preserving Optic Nerve Transection in Rats to Assess Axon Regeneration and Interventions Targeting the Retinal Ganglion Cell Axon
}

\author{
Jiun L. Do ${ }^{1}$, Salam Allahwerdy ${ }^{1}$, Ryan C. David ${ }^{1}$, Robert N. Weinreb ${ }^{1}$, Derek S. Welsbie ${ }^{1}$ \\ ${ }^{1}$ Hamilton Glaucoma Center, Viterbi Family Department of Ophthalmology, Shiley Eye Institute, University of California San Diego
}

\section{Corresponding Author}

Jiun L. Do

jiundo@health.ucsd.edu

\section{Citation}

Do, J.L., Allahwerdy, S., David, R.C., Weinreb, R.N., Welsbie, D.S. SheathPreserving Optic Nerve Transection in Rats to Assess Axon Regeneration and Interventions Targeting the Retinal Ganglion Cell Axon. J. Vis. Exp. (163), e61748, doi:10.3791/61748 (2020).

\section{Date Published}

September 6, 2020

DOI

$10.3791 / 61748$

URL

jove.com/video/61748

\section{Abstract}

Retinal ganglion cell (RGC) axons converge at the optic nerve head to convey visual information from the retina to the brain. Pathologies such as glaucoma, trauma, and ischemic optic neuropathies injure RGC axons, disrupt transmission of visual stimuli, and cause vision loss. Animal models simulating RGC axon injury include optic nerve crush and transection paradigms. Each of these models has inherent advantages and disadvantages. An optic nerve crush is generally less severe than a transection and can be used to assay axon regeneration across the lesion site. However, differences in crush force and duration can affect tissue responses, resulting in variable reproducibility and lesion completeness. With optic nerve transection, there is a severe and reproducible injury that completely lesions all axons. However, transecting the optic nerve dramatically alters the blood brain barrier by violating the optic nerve sheath, exposing the optic nerve to the peripheral environment. Moreover, regeneration beyond a transection site cannot be assessed without reapposing the cut nerve ends. Furthermore, distinct degenerative changes and cellular pathways are activated by either a crush or transection injury.

The method described here incorporates the advantages of both optic nerve crush and transection models while mitigating the disadvantages. Hydrostatic pressure delivered into the optic nerve by microinjection completely transects the optic nerve while maintaining the integrity of the optic nerve sheath. The transected optic nerve ends are reapposed to allow for axon regeneration assays. A potential limitation of this method is the inability to visualize the complete transection, a potential source of variability. However, visual confirmation that the visible portion of the optic nerve has been transected is indicative of a complete optic nerve transection with $90-95 \%$ success. 
This method could be applied to assess axon regeneration promoting strategies in a transection model or investigate interventions that target the axonal compartments.

\section{Introduction}

Axonal injury and degeneration occur in retinal ganglion cells (RGCs) following trauma or in neurodegenerative diseases such as glaucoma ${ }^{1,2}$. The loss of RGCs and disruption of retinofugal projections result in permanent vision loss ${ }^{3}$. To understand the molecular pathways responsible for the degenerative processes and to develop strategies to mitigate axonal and RGC loss or to regenerate RGC axons, experimental animal models have been used to simulate optic nerve injury, including optic nerve crush and optic nerve transection models. In selecting an experimental model, one must account for the advantages and disadvantages of each approach as well as the molecular pathways activated by the injury ${ }^{4}$.

The rationale for developing the method described here is to leverage the advantages of optic nerve crush $^{5}$ and transection ${ }^{6}$ models while mitigating the disadvantages. The objectives of this method were to generate a reproducible optic nerve injury in which all axons are unquestionably and completely transected, exposure to the peripheral immune system is minimized, and the transected ends of the optic nerve are easily reapposed to allow for the evaluation of RGC regeneration. Additionally, the method was developed to allow for compartmentalized access to the axonal portion of injured RGCs and to deliver axon specific interventions (e.g., neurotrophic factors, cellular transplants) locally to the retroorbital optic nerve.

There are multiple advantages of this technique over alternative methods. Compared to an optic nerve crush, this method completely and reliably transects the optic nerve; this addresses a potential issue of undesirable axon sparing ${ }^{7}$. Additionally, the described method causes a severe axonal injury that is not dependent on the amount and duration of force applied by the operator as in a crush injury, thereby reducing variability ${ }^{8}$. In contrast to established methods of transecting the optic nerve, the approach detailed in this protocol maintains the integrity of the optic nerve sheath. An advantage of preserving the optic nerve sheath is that it prevents the optic nerve from being exposed to the peripheral immune system. Furthermore, the mechanical forces exerted by the optic nerve sheath on the transected optic nerve reappose the cut nerve ends without the need for challenging microsurgical manipulations ${ }^{9}, 10,11$. Finally, with the optic nerve sheath intact, the method produces a physical space between the optic nerve stumps into which stem cells, neurotrophic factors, or polymers can be introduced to lesioned RGC axons directly.

The optic nerve crush is the gold standard model in which optic nerve regeneration strategies are assessed to determine the effectiveness of treatments. The size of the rodent optic nerve limits the possible manipulations, especially the transection and re-adaptation of the nerve. However, in the field of spinal cord injury and regeneration, there is consensus that a complete transection is the ideal model to distinguish axonal regeneration from spared axon $^{12}$. The method described here reduces the technical barriers to assess regenerative strategies in an optic nerve transection model. As such, this model could be used to validate promising strategies identified in optic nerve crush 
paradigms with an optic nerve transection. Additionally, as this model directly targets the axonal compartment, it enables studies of interventions on injured adult RGC axons and the mechanisms responsible for axonal degenerative and regenerative processes.

The model of optic nerve transection described in this study completely transects the optic nerve while preserving the optic nerve sheath. This novel approach is appropriate for experiments that aim to assess axon regeneration in a transection model without the need for the technically challenging process of reapposing the optic nerve ends. Aspects of the technique are similar to performing an optic nerve crush; therefore, the approach can be performed by operators experienced with an optic nerve crush. The surgical approach does not require specially designed instruments and can be completed with readily available surgical instruments and a microinjection system, making it accessible and economical.

\section{Protocol}

Procedures involving animals were approved by the Institutional Animal Care and Use Committee (IACUC) of the Veterans Affairs San Diego Healthcare System. Surgical instruments and solutions were sterilized prior to surgery to limit postoperative infections and complications.

\section{Surgical technique}

1. Conduct experiments using aseptic techniques and according to institution specific animal use protocols.

2. Sterilize instruments and materials that contact living tissues to prevent infections, avoid negatively impacting animal welfare, and minimize potential negative influences on the study. Clean surgical instruments thoroughly with water and detergent or enzymatic products to remove foreign materials and sterilize by steam or flash sterilization.

3. Aliquot liquids into sterile containers in a tissue culture hood. Sterilize the microliter syringe by flushing it with $70 \%$ ethanol and rinsing it with sterile phosphate buffered saline (PBS).

\section{Anesthesia}

1. Anesthetize rats using an isoflurane vaporizer system. Vaporize isoflurane at a concentration of $4.5 \%$ using medical grade oxygen at a rate of $1 \mathrm{~L} / \mathrm{min}$ into an attached anesthesia box. Place the animal into the anesthesia box until breathing slows and the animal is sedated.

NOTE: This initial step using anesthesia with a gas anesthetic is not necessary if the operator is comfortable and familiar with administering injectables anesthetics to non-sedated animals.

2. Withdraw a sufficient volume of an anesthetic cocktail composed of ketamine $(50 \mathrm{mg} / \mathrm{kg})$, xylazine $(2.6 \mathrm{mg} /$ $\mathrm{kg})$, and acepromazine $(0.5 \mathrm{mg} / \mathrm{kg})$ into a $30 \mathrm{G}$ tuberculin syringe. Remove the sedated animal from the anesthesia box. Administer the injection of anesthesia intraperitoneally to attain consistent depth of sedation throughout the procedure and return the animal to its cage.

3. Begin the procedure when a toe pinch fails to evoke a response. Evaluate the animal for depth and rate of breathing and with a toe pinch every 5 min throughout the procedure to ensure the absence of pain.

4. Upon completion of surgery, remove the animal from the stereotaxic frame. Administer a subcutaneous injection of saline (3 mL), ampicillin (0.15-0.2 mg/kg), and banamine 
(2.5-5 $\mathrm{mg} / \mathrm{kg}$ ) to provide analgesia and fluid support. Transfer the animal to a heated incubator to maintain body temperature.

\section{Surgical approach}

1. Place the rat (7-8 weeks of age, Fischer 344 strain) in a head stereotaxic frame and on top of a heated pad. Position the stereotaxic frame with the rat head facing the surgeon's left and center the left orbit in the surgical field of view.

2. To the left eye apply a drop of proparacaine and clean the eye by applying $5 \%$ povidone-iodine to the eyelid and the eye. Spread ophthalmic ointment on the cornea of both eyes to maintain corneal moisture and prevent cataract formation.

3. Pass a 4-0 polyglactin suture through the epidermis of the upper eyelid to the central aspect of the eyelid margin, using this suture for a temporary tarsorrhaphy in later steps. Use the suture to evert the eyelid and expose the superior fornix.

4. Tape the polyglactin suture to the stereotaxic frame to apply constant traction and exposure. Avoid excessive traction as this can limit exposure of the retroorbital contents.

5. Using Colibri forceps, elevate the superior conjunctiva and incise the conjunctiva along the limbus with a pair of Vannas scissors. Make a 4 o'clock peritomy along the superior limbus.

6. Apply slight inferior traction to the globe by placing a looped polypropylene suture along the superior limbus with the free ends below the globe. Residual tissue along the superior limbus following the peritomy is sufficient to provide traction on the suture loop without needing to pass the suture through the sclera or limbus.

1. Secure the ends of the loop with a bulldog clamp and allow the clamp to dangle freely in midair. The weight of the clamp will apply inferior traction on the globe and expose more of the retroorbital space.

7. Blunt dissect with Vannas scissors along the sclera following the posterior aspect of the globe and under the superior rectus. Disinsert the superior rectus muscles from the globe by cutting the muscle tendon with Vannas scissors to access the retroorbital space.

\section{Accessing the optic nerve}

1. Blunt dissect with Dumont $\# 5 / 45$ forceps along the temporal aspect of the posterior globe and identify the superior lateral vortex vein. Further blunt dissect nasal to the superior lateral vortex vein to expose the orbital muscle cone around the optic nerve and avoid prematurely damaging the optic nerve. Avoid injuring the vortex vein as this can cause significant bleeding. If bleeding occurs, a cotton tip applicator can be applied until the bleeding stops.

2. Using the Dumont \#5/45 forceps, carefully insert the tip into the temporal aspect of the orbital muscle cone and open the forceps parallel to the course of the muscle fibers to reveal the optic nerve. Use the forceps to laterally displace the muscle fibers overlying the optic nerve and fully expose the nerve. Ensure that only the optic nerve sheath is covering the optic nerve as residual muscle fibers will prevent the pulled glass capillary pipette from easily piercing into the optic nerve.

3. Maintain exposure of the nerve by placing two Dumont \#5/45 forceps along both lateral aspects of the nerve 
and opening the forceps. The amount of exposure should allow for the optic nerve to be crushed 1.5-2.0 mm posterior to the globe and for a pulled glass capillary pipette to be inserted into the optic nerve from the dorsal aspect.

\section{Transecting the optic nerve inside the optic sheath}

1. Using Dumont \#5/45 forceps, place the tips on either side of the optic nerve at least $1.5-2.0 \mathrm{~mm}$ posterior to the globe. Ensure the tips of the forceps span the diameter of the nerve. Completely close the forceps for $5 \mathrm{~s}$ to crush the optic nerve and make note of the position of the crush site.

2. With a microliter syringe, fill a glass pulled capillary pipette with 1-2 $\mu \mathrm{L}$ of PBS and attach the pipette to a pipette holder. Mount the pipette holder to a micromanipulator and attach the micromanipulator onto the stereotaxic frame.

3. Adjust the microinjection system to deliver a single pulse of 4 ms duration at a pressure of 20 psi with each push of the button. Position the pipette into the surgical field.

4. Under the surgical scope, use a pair of \#55 forceps to break and bevel the pipette tip to a size $(\sim 20-30 \mu \mathrm{m}$ diameter) capable of delivering a small volume but large enough to provide sufficient rigidity to penetrate the optic nerve sheath. Confirm patency of the pipette tip by giving a single pulse and observing for fluid at the pipette tip.

5. Refine the position of the pipette tip to just above the site of the optic nerve crush in the center of the optic nerve and in contact with the optic nerve sheath. Note the vertical position of the pipette on the micromanipulator to later determine a depth of $200 \mu \mathrm{m}$.
6. Lower the pipette while observing the tip under the surgical scope until the pipette enters the optic nerve. If the pipette tip bends and lacks the rigidity to penetrate the optic nerve sheath, retract the pipette and use a pair of \#55 forceps to decrease the length and increase the diameter of the pipette tip. Once adjustments to the pipette tip have been made, reattempt to penetrate the optic nerve sheath with the pipette.

7. Upon entry into the optic nerve with the pipette tip, retract the pipette if necessary. The position of the pipette tip, as indicated by the stereotaxic micromanipulator, should be $200 \mu \mathrm{m}$ below the surface of the optic nerve from the initial position noted in step 5.5 .

8. While observing the optic nerve under the surgical scope, apply pulses of hydrostatic pressure with the microinjection system. As pulses are applied, a linear separation between the distal and proximal ends of the optic nerve should be noted to verify transection of the optic nerve.

NOTE: Injection volume of $250-500 \mathrm{~nL}$ is generally sufficient to provide the force necessary to transect the optic nerve. Injections requiring more than $1 \mu \mathrm{L}$ may indicate a need to reposition the injection site. Larger volumes injected into the intact parenchyma may not cause additional RGC damage given the method completely transects the optic nerve but is more likely to track along the fascicles of the optic nerve. Failure to observe a linear transection of the optic nerve despite injection of a sufficient fluid volume likely indicates mispositioning of the pipette at the crush site and a need for repositioning. A $50 \%$ increase in the injection pressure may also be necessary. 


\section{Closing and recovery}

1. Retract the pipette and remove the retracting Dumont \#5/45 forceps. Return the eye to a neutral position by removing the bulldog clamp and looped polypropylene suture from the globe.

2. Reposition the conjunctiva to the corneal limbus, insuring to release any conjunctiva that has inverted to allow for proper apposition of the tissue and healing. Apply ophthalmic topical antibiotic ointment to the eye.

3. Remove the tape from the eyelid $4-0$ polyglactin suture and maintain the suture in place to use for a temporary tarsorrhaphy. Pass the suture through the eyelid margin of the lower eyelid, through the subcuticular area inferiorly, and exiting through the epidermis. Tie the suture with just enough pressure to close the eye.

4. Administer postsurgical analgesics according to institutional protocols and animal care authority guidelines. House the animal independently in a heated cage to recover after surgery. Do not place bedding in the recovery cage to prevent accidental aspiration.

\section{Representative Results}

Transection of the optic nerve typically results in the apoptotic loss of $80-90 \%$ of injured RGCs within 14 days after injury.
The described technique transects the optic nerve while maintaining the optic nerve sheath integrity (Figure 1). The degree of RGC loss is comparable to traditional optic nerve transection and optic nerve crush models with the advantage that the cut nerve ends are effortlessly apposed after transection with the method described here (Figure 2). Reconnecting the cut optic nerve ends in this manner enables evaluation of RGC axonal regeneration in a transection model by providing a substrate on which axons may grow and without the need for microsurgical manipulation to reconnect the cut nerve ends (Figure 3). Anterograde tracing of RGC axons with cholera toxin B subunit (CTB) demonstrates that CTB positive axons are completely transected following a sheath preserving optic nerve transection (Figure 4). Preserving the optic nerve sheath while transecting the optic nerve also creates an enclosed space into which investigative materials, such as neurotrophic factors or cells, can be delivered to lesioned RGC axons and maintained in position (Figure 5). During initial phases of training, there is an expected $60-70 \%$ success rate of total transection. With experience, the success rate of total transection is approximately $90-95 \%$. 

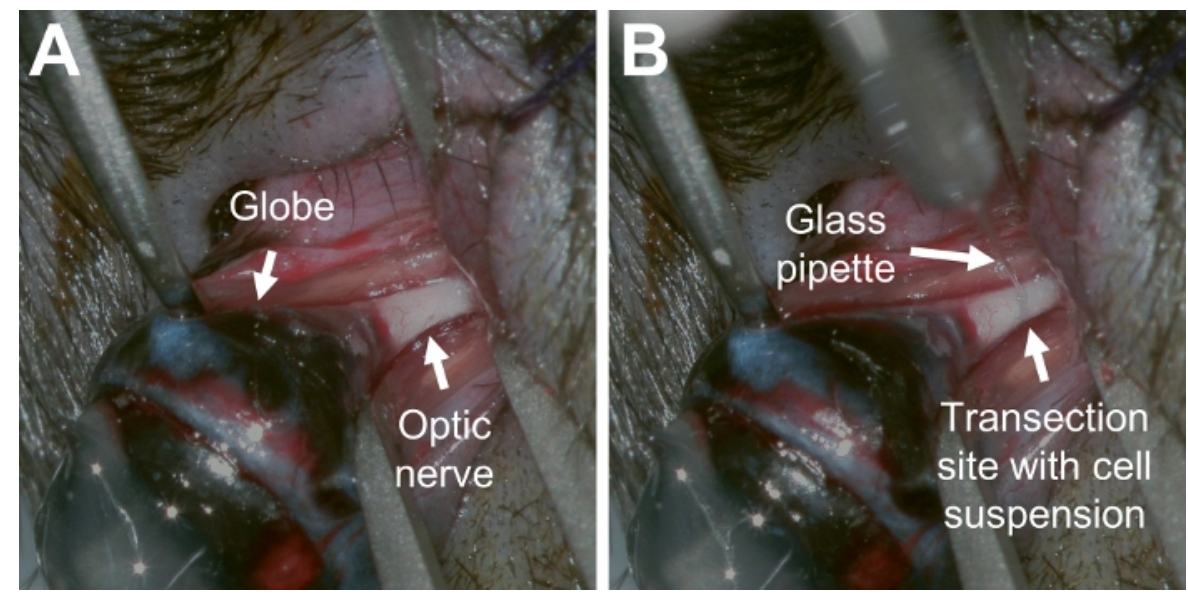

Figure 1: Transecting the optic nerve while preserving the optic nerve sheath. (A) Picture of the surgical field demonstrating exposure of the intact optic nerve prior to transection. (B) Picture of the optic nerve following transection. A fine glass pipette pierced the optic nerve sheath at the transection site and delivered a cell suspension (turbid solution) into the space between the optic nerve ends. Please click here to view a larger version of this figure.
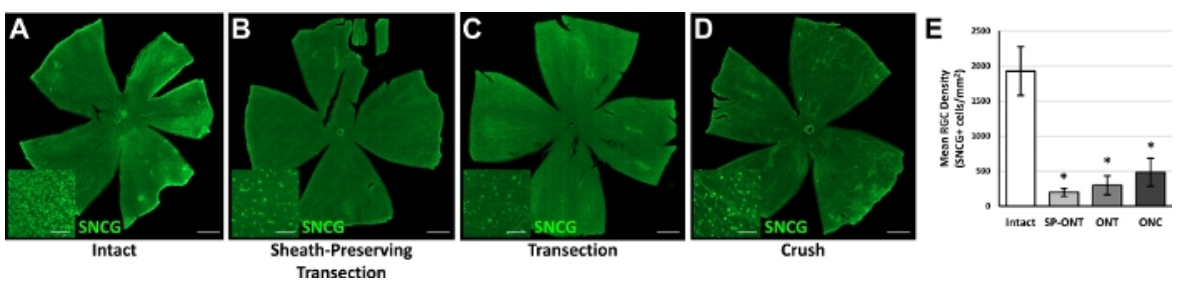

\section{Figure 2: Retinal ganglion cell loss following an optic nerve sheath preserving optic nerve}

transection. Representative whole retina flat mounts from eyes with $(\mathbf{A})$ an intact optic nerve, $(\mathbf{B})$ an optic nerve sheath preserving optic nerve transection, (C) a traditional optic nerve transection, or (D) optic nerve crush 14 days prior were immunolabeled for gamma synuclein (SNCG). Images were obtained from a fluorescence microscope using a 10x objective, corrected for shading, and stitched to generate a single image. The loss of retinal ganglion cell (RGC) bodies throughout the retina was apparent in lesioned eyes. Insets show higher magnification images of the retina and demonstrate significant loss of RGCs following optic nerve injuries. (E) Quantification of RGC survival demonstrates significant RGC loss following optic nerve injuries compared to control intact optic nerves. ${ }^{*} p<0.05$ compared to intact; one-way ANOVA with post-hoc Tukey test. $n=3$ animals per group; error bars represent SD. SP-ONT, sheath-preserving optic nerve transection; ONT, optic nerve transection; ONC, optic nerve crush. Scale bars $=1,000 \mu \mathrm{m}$. Scale bars in insets $=100 \mu \mathrm{m}$. Please click here to view a larger version of this figure. 

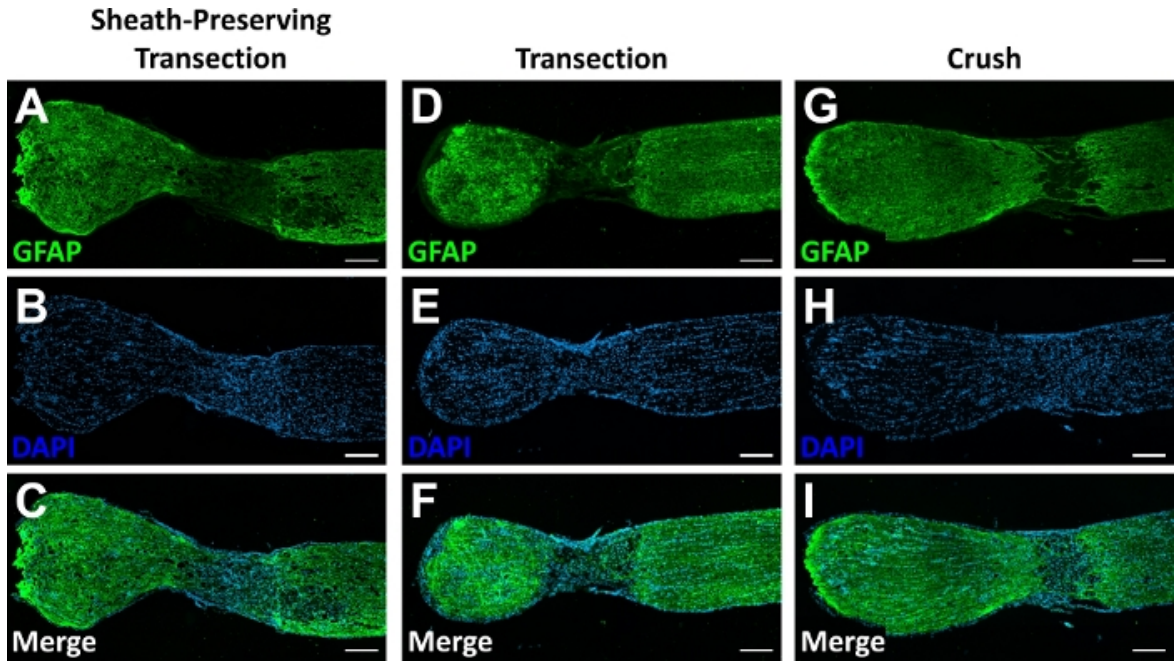

Figure 3: Sections of optic nerves following optic nerve injuries. Representative images of longitudinal section through optic nerves 14 days after (A-C) an optic nerve sheath preserving transection, (D-F) traditional optic nerve transection, or (GI) optic nerve crush. $(\mathbf{A}, \mathbf{D}, \mathbf{G})$ Immunolabeling for glial fibrillary acidic protein (GFAP) delineates the extent of the lesion while (B,E,H) DNA labeling with 4',6-diamidino-2-phenylindole (DAPI) demonstrates contiguous cellularity along the length of the optic nerve and within the lesion site. (C,F,I) Merged images demonstrating localization of GFAP-positive neural tissue and cellularity at the lesion site. Scale bars $=200 \mu \mathrm{m}$. Please click here to view a larger version of this figure. 

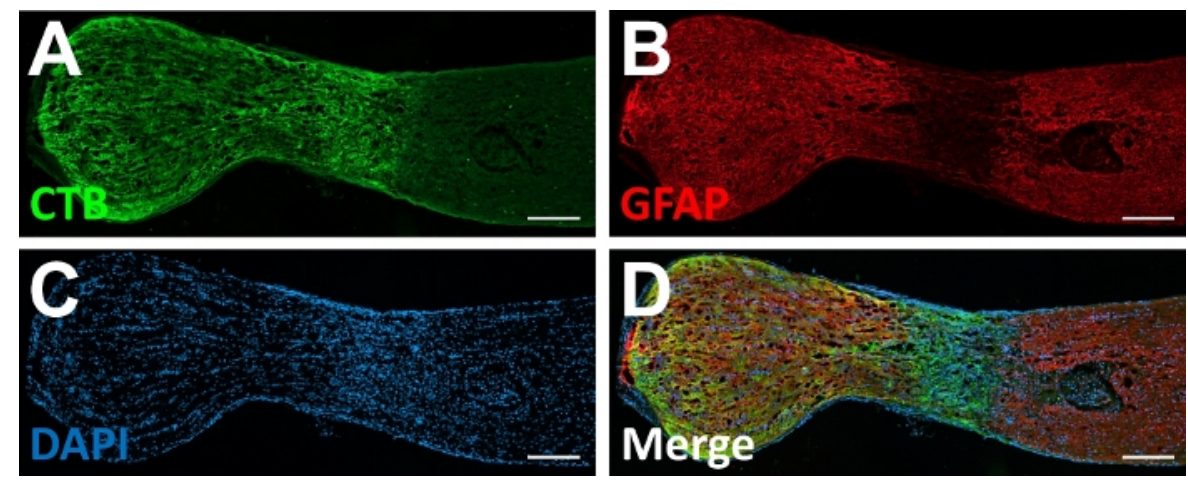

Figure 4: Section of an optic nerve following an optic nerve sheath preserving optic nerve

transection. Representative images of a longitudinal section through an optic nerve 14 days after an optic nerve sheath preserving transection with anterograde axon tracing with an intravitreal cholera toxin B subunit (CTB) injection. (A) Complete lesioning of CTB labeled RGC axons extending from the globe (left) toward the brain (right) can be observed. (B) Immunolabeling for glial fibrillary acidic protein (GFAP) delineates an extensive lesion that involves the entire diameter of the optic nerve. (C) DNA labeling with 4',6-diamidino-2-phenylindole (DAPI) demonstrates cellularity within the lesion site. (D) A merged image demonstrating localization of CTB-labeled axons, GFAP-positive neural tissue, and cellularity at the lesion site. Scale bars $=200 \mu \mathrm{m}$. Please click here to view a larger version of this figure.
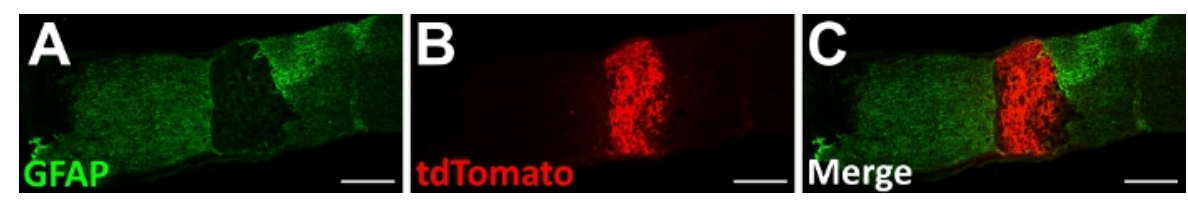

Figure 5: Longitudinal section of a transected optic nerve that received a cell graft. Representative images of a longitudinal section through an optic nerve 14 days after an optic nerve sheath preserving transection and transplantation of neural stem cells (NSCs) expressing the fluorescent protein tdTomato. (A) Immunolabeling for glial fibrillary acidic protein (GFAP) demonstrated complete separation of the transected optic nerve ends. (B) NSCs expressing tdTomato were contained within the space created by the described technique and continued to survive following transplantation. (C) Grafted NSCs were directly apposed to both cut ends of the transected optic nerve. Scale bars, $200 \mu \mathrm{m}$. Please click here to view a larger version of this figure. 


\section{Discussion}

Surgical procedures describing the optic nerve transection model have been published previously ${ }^{6}$. However, the techniques detailed in those protocols involve incising the meningeal sheath to transect the optic nerve. Furthermore, in order to assess RGC axon regeneration in previous transection models, challenging microsurgical manipulations were required to either appose the cut optic nerve ends or a peripheral nerve graft to the proximal optic nerve stump ${ }^{10,13}$. The protocol described here minimally disrupts the optic nerve sheath while transecting the optic nerve and allows for evaluations of $R G C$ axon regeneration in a transection model without the need for technically challenging microsurgical manipulations.

Several steps are critical in this protocol. Care should be taken to avoid damaging the ophthalmic artery and the vasculature supplying the optic nerve head. Therefore, step 5.1 should be completed at least $1.5-2.0 \mathrm{~mm}$ posterior to the globe. If damage to the ophthalmic artery occurs and disrupts the retinal blood supply, the eye should be excluded from further experiments as phthisis is likely to follow. During steps 5.4-5.6, it is important to maintain the integrity of the optic nerve sheath and minimize the size of the opening through which the glass pipette enters the optic nerve. Doing so forms a tight seal around the pipette tip to reduce fluid reflux and allows the generation of sufficient hydrostatic pressure to transect the optic nerve. Beveling the tip of the glass pipettes will improve the ease with which the pipette enters the optic nerve without causing collateral damage.

There are potential modifications that operators could make to improve the accessibility of this method. The described procedures involve minimal dissection and removal of orbital tissue with preservation of the facial and trigeminal nerve. While this reduces morbidity and risks of bleeding, tissues such as orbital fat and the lacrimal gland may limit visualization of crucial structures. Careful removal of tissue obstructing the surgical field may be necessary to enhance visualization, especially in older animals. A lateral approach could also be used to improve access to the optic nerve. However, a lateral dissection risks damage to additional structures including the trigeminal nerve, is more involved, and may present its own challenges to directing instrumentation for injections.

A possible limitation of this method is the inability to directly manipulate the optic nerve and completely visualize the entire transection. Therefore, there is the possibility of an incomplete transection. However, we have observed that visual confirmation of the nerve ends separating during step 5.8 is a reliable indicator of a successful and complete transection. Should the nerve ends fail to separate, repositioning the injection pipette or increasing the pressure of the injection by $50 \%$ should provide sufficient force to completely transect the nerve.

With respect to existing methods, this approach preserves the integrity of the optic nerve sheath. In maintaining the integrity of the optic nerve sheath, the ends of the transected optic nerve are not exposed to the orbital environment and peripheral immune system, thereby limiting exposure to immune factors that could potentially influence RGC responses. Additionally, preserving the integrity of the optic nerve sheath while transecting the optic nerve creates an enclosed physical space bounded by the optic nerve ends and the optic nerve sheath. Localized delivery of neurotrophic factors, cells, or polymers to the axonal compartment of the injured RGCs can be achieved by injecting into the newly formed space. ${ }^{14}$ Alternatively, RGC axon regeneration 
can be evaluated in a transection model by allowing the transected optic nerve ends to anastomose without the need for challenging microsurgical techniques.

Applications of this method include the evaluation of the injured RGC axonal compartment with axon-specific interventions to identify pathways responsible for axonal degeneration and prevent axonal loss following a transection injury. Furthermore, this method makes studies of RGC axonal regeneration in a transection model accessible to the broader research community by removing the need for technically difficult optic nerve anastomoses procedures. Interventions aiming to promote RGC axon regeneration could be evaluated with this severe injury model and provide consistent and reproducible results without the concern for spared axons.

\section{Disclosures}

The authors have no competing or conflicts of interest to disclose.

\section{Acknowledgments}

This works was supported in part by a K12 Career Development grant (5K12EY024225-04, National Eye Institute), a P30 core grant (P30EY022589, National Eye Institute), a Mentoring for the Advancement of Physician Scientists award (American Glaucoma Society), and an unrestricted grant from Research to Prevent Blindness (New York, NY).

\section{References}

1. Quigley, H. A. Optic Nerve Damage in Human Glaucoma. Archives of Ophthalmology. 99 (4), 635 (1981).
2. Steinsapir, K. D., Goldberg, R. A. Traumatic optic neuropathy: An evolving understanding. American Journal of Ophthalmology. 151 (6), 928-933 (2011).

3. Kerrigan-Baumrind, L. A., Quigley, H. A., Pease, M. E., Kerrigan, D. F., Mitchell, R. S.. Number of ganglion cells in glaucoma eyes compared with threshold visual field tests in the same persons. Investigative Ophthalmology and Visual Sciences. 41 (3), 741-748 (2000).

4. Agudo, M. et al. Time course profiling of the retinal transcriptome after optic nerve transection and optic nerve crush. Molecular Visison. 14 (June), 1050-1063 (2008).

5. Cameron, E., Xia, X., Galvao, J., Ashouri, M., Kapiloff, M., Goldberg, J.. Optic nerve crush in mice to study retinal ganglion cell survival and regeneration. Bio-Protocol. 10 (6), 139-148 (2020).

6. Magharious, M. M., D'Onofrio, P. M., Koeberle, P. D. Optic nerve transection: A model of adult neuron apoptosis in the central nervous system. Journal of Visualized Experiments. (51), e2241 (2011).

7. Fischer, D., Harvey, A. R., Pernet, V., Lemmon, V. P., Park, K. K. Optic nerve regeneration in mammals: Regenerated or spared axons? Experimental Neurology. 296, 83-88 (2017).

8. Kim, J., Sajid, M. S., Trakhtenberg, E. F. The extent of extra-axonal tissue damage determines the levels of CSPG upregulation and the success of experimental axon regeneration in the CNS. Science Report. 8 (1), 1-10 (2018).

9. Inoue, T., Hosokawa. M., Morigiwa, K., Ohashi, Y., Fukuda, Y. Bcl-2 overexpression does not enhance in vivo axonal regeneration of retinal ganglion cells after 
peripheral nerve transplantation in adult mice. Journal of Neuroscience. 22 (11), 4468-4477 (2002).

10. Fischer, D., Heiduschka, P., Thanos, S. Lens-injurystimulated axonal regeneration throughout the optic pathway of adult rats. Experimental Neurology. 172 (2), 257-272 (2001).

11. Cui, Q., Harvey, A. R. CNTF promotes the regrowth of retinal ganglion cell axons into murine peripheral nerve grafts. Neuroreport. 11 (18), 3999-4002 (2000).

12. Tuszynski, M. H., Steward, O. Concepts and methods for the study of axonal regeneration in the CNS. Neuron. $\mathbf{7 4}$ (5), 777-791 (2012).

13. You, S. W. et al. Large-scale reconstitution of a retinato-brain pathway in adult rats using gene therapy and bridging grafts: An anatomical and behavioral analysis. Experimental Neurology. 279, 197-211 (2016).

14. Wang, D. et al. Localized co-delivery of CNTF and FK506 using a thermosensitive hydrogel for retina ganglion cells protection after traumatic optic nerve injury. Drug Delivery. 27 (1), 556-564 (2020). 